\title{
ARTíCULO \\ Crecimiento, madurez y mortalidad del pez gallo, Callorhinchus callorynchus, en el Golfo San Matías, Patagonia norte, Argentina
}

\author{
Growth, maturity and mortality of cockfish, Callorhinchus callorynchus, in \\ San Matías Gulf, Northern Patagonia, Argentina \\ Juan Bernasconi ${ }^{1,2}$, Luis Cubillos ${ }^{3}$, Enzo Acuña ${ }^{4,5}$, \\ Raquel Perier ${ }^{1,6}$ y Edgardo Di Giácomo ${ }^{1,6}$
}

\begin{abstract}
${ }^{1}$ Grupo de Estudio de Peces Cartilaginosos (CONDROS), Instituto de Biología Marina y Pesquera Almirante Storni, Guemes 1030, San Antonio Oeste, Argentina. jfbernasconi@yahoo.com.ar

${ }^{2}$ Consejo Nacional de Investigaciones Científicas y Técnicas (CONICET), Rivadavia 1917, Ciudad Autónoma de Buenos Aires, Argentina

${ }^{3}$ Laboratorio de Ecología de Poblaciones Marinas (EPOMAR) y Programa COPAS Sur-Austral, Departamento de Oceanografía, Universidad de Concepción, Casilla 160-C, Concepción, Chile

${ }^{4}$ Departamento de Biología Marina, Universidad Católica del Norte, Sede Coquimbo, Casilla 117, Coquimbo, Chile

${ }_{5}^{5}$ Núcleo Milenio de Ecología y Manejo Sustentable de Islas Oceánicas (Ecology and Sustainable Management of Oceanic Islands - ESMOI)

${ }^{6}$ Universidad Nacional del Comahue, Guemes 1030, San Antonio Oeste, Argentina
\end{abstract}

\begin{abstract}
Detailed knowledge of the life history parameters of chondrichthyans is required to assess their population status and vulnerability to fishing mortality. The objective of this study was to estimate growth, maturity length $\left(\mathrm{LM}_{50 \%}\right)$, longevity $\left(\mathrm{E}_{\max }\right)$ and natural mortality rate $(\mathrm{M})$ of the cockfish, Callorhinchus callorynchus, in the San Matías Gulf, Argentina. The length range in the commercial catches was 22 to $58 \mathrm{~cm}$ for males and 25 a $76 \mathrm{~cm}$ for females, while in fisheries surveys was 13 to $59 \mathrm{~cm}$ for males and 14 to $69 \mathrm{~cm}$ for females. The sex ratio was biased to the males. The Gompertz L0 fixed growth model was the most adequate according to biological selection criteria. The parameters were: i) females: $L_{\infty}=70.75 \mathrm{~cm}$ and $\mathrm{K}=0.17$ year $^{-1}$; and ii) males: $\mathrm{L}_{\infty}=56.11 \mathrm{~cm}$ and $\mathrm{K}=0.257 \mathrm{year}^{-1} . \mathrm{A} \mathrm{LM}_{50 \%}=43.39 \mathrm{~cm}$, a $\mathrm{E}_{\max }=13.7$ year and a $\mathrm{M}=0.35$ year ${ }^{-1}$ were estimated for males, while a $\mathrm{LM}_{50 \%}=47.48 \mathrm{~cm}$, a $\mathrm{E}_{\max }=21.4$ year and a $\mathrm{M}=0.23$ year $^{-1}$ were estimated for females. The results of this work show that there is sexual dimorphism in life-history characteristics of the cockfish. Females reach a larger size, mature later, grow slower and live longer than males.
\end{abstract}

Key words: Cockfish, growth, maturity, mortality, Chondrichthyans

Resumen.- Conocer los parámetros de historia de vida de las especies de condrictios es uno de los principales requisitos para evaluar el estado de sus poblaciones y vulnerabilidad a la explotación pesquera. En este estudio se estimó el crecimiento en longitud, la talla de madurez $\left(\mathrm{LM}_{50 \%}\right)$, la longevidad $\left(\mathrm{E}_{\max }\right)$ y la tasa de mortalidad natural (M) del pez gallo, Callorhinchus callorynchus, en el Golfo San Matías, Argentina. En los muestreos de las capturas comerciales se obtuvo un rango de tallas de 22 a $58 \mathrm{~cm}$ para machos y de 25 a $76 \mathrm{~cm}$ para las hembras, mientras que en las campañas de investigación fue de 13 a $59 \mathrm{~cm}$ para los machos y de 14 a $69 \mathrm{~cm}$ para las hembras. La proporción de sexos estuvo sesgada hacia los machos. El modelo de crecimiento de Gompertz LO fijo fue el más adecuado según el criterio biológico de selección. Los parámetros fueron: i) hembras: $L_{\infty}=70,75 \mathrm{~cm}$ y $K=0,17$ año-1 ${ }^{-1}$ ii) machos: $L_{\infty}=56,11 \mathrm{~cm}$ y $K=0,257$ año $^{-1}$. Para machos se obtuvo un $\mathrm{LM}_{50 \%}=43,39 \mathrm{~cm}$, una $E_{\max }=13,7$ años y una $M_{\text {media }}=0,35$ año-1 y para hembras se obtuvo un $\mathrm{LM}_{50 \%}=47,48 \mathrm{~cm}$, una $E_{\max }=21,4$ años y una $M_{\text {media }}=0,23$ año $^{-1}$. Los resultados de este estudio indican que existe dimorfismo sexual en la especie, siendo las hembras las que alcanzan mayor talla, maduran más tarde, crecen más lento y son más longevas que los machos.

Palabras clave: Pez gallo, crecimiento, madurez, mortalidad, Condrictios

\section{INTRODUCCIÓN}

El 'pez gallo' o 'pejegallo', Callorhinchus callorynchus (Linnaeus, 1758), es un pez cartilaginoso (clase Chondrichthyes) perteneciente al grupo de los holocéfalos (Subclase: Holocephali). Esta especie presenta una amplia distribución en América del Sur. En el Océano Pacífico se encuentra desde el norte de Perú hasta el Estrecho de Magallanes en Chile (Chirichigno \& Cornejo 2001), mientras que en el Océano Atlántico se 
distribuye desde el estado brasileño de São Paulo (Figuereido 1977), extendiéndose por las costas de Uruguay y Argentina, hasta el Canal de Beagle (López et al. 2000). En ambos océanos el pez gallo representa un recurso importante tanto para pescadores artesanales como industriales (Di Giácomo \& Perier 1991, Alarcón et al. 2011).

Los desembarques de pez gallo en Argentina representan aproximadamente el $97 \%$ de las capturas totales de la especie en el Atlántico Sudoccidental y desde 1992 hasta el 2011 presentaron una tendencia creciente. El desembarque mínimo se registró en 1992, con 490 ton y el máximo en el 2009, con 2780 ton, con un promedio anual de 1600 ton para el período 1992-2011 (Sánchez et al. 2012). La pesca comercial del pez gallo en Argentina se produce principalmente como bycatch en pesquerías de arrastre que tienen como especie blanco a la merluza común, Merluccius hubbsi, o a especies costeras como la corvina (Micropogonias furnieri) y la pescadilla (Cynoscion guatucupa). Los estratos de buques con eslora menor a los $19 \mathrm{~m}$ o entre los 19-28 m son los que principalmente capturan pez gallo (Sánchez et al. 2011).
Uno de los principales núcleos costeros de la especie se encuentra en el Golfo San Matías (GSM) $\left(41^{\circ}-42^{\circ} \mathrm{S}, 64^{\circ}-\right.$ $65^{\circ} \mathrm{O}$ ) (Fig. 1), donde se identifican zonas de alimentación, de reproducción y de crianza de la especie (Di Giácomo 1992). En esta región, las capturas comerciales se producen como by-catch por la flota de arrastre demersal que opera en el puerto de San Antonio Oeste (provincia de Río Negro) y tiene como especie objetivo la merluza común, Merluccius hubbsi. Aproximadamente 15 buques operan en el GSM con un tamaño de eslora entre los 12 y $38 \mathrm{~m}$. Según el registro de la estadística provincial, desde 1986 hasta 2011 en el GSM el promedio anual de capturas de pez gallo fue de 490 ton, registrándose una tendencia de incremento durante el período, con un mínimo de 100 ton en 1987 y un máximo de 1450 ton en el $2009^{1}$. Para esta pesquería, el pez gallo representa un recurso económico importante dado que históricamente es la segunda especie en toneladas desembarcadas y las capturas comerciales se registran durante todo el año (Di Giácomo \& Perier 1991, Di Giácomo \& Perier 2008) ${ }^{1}$. A su vez, en esta región el pez gallo es la principal especie entre los peces cartilaginosos capturados por by-catch en cuanto a volumen de captura y frecuencia de ocurrencia en los viajes de pesca (Perier et al. 2011).

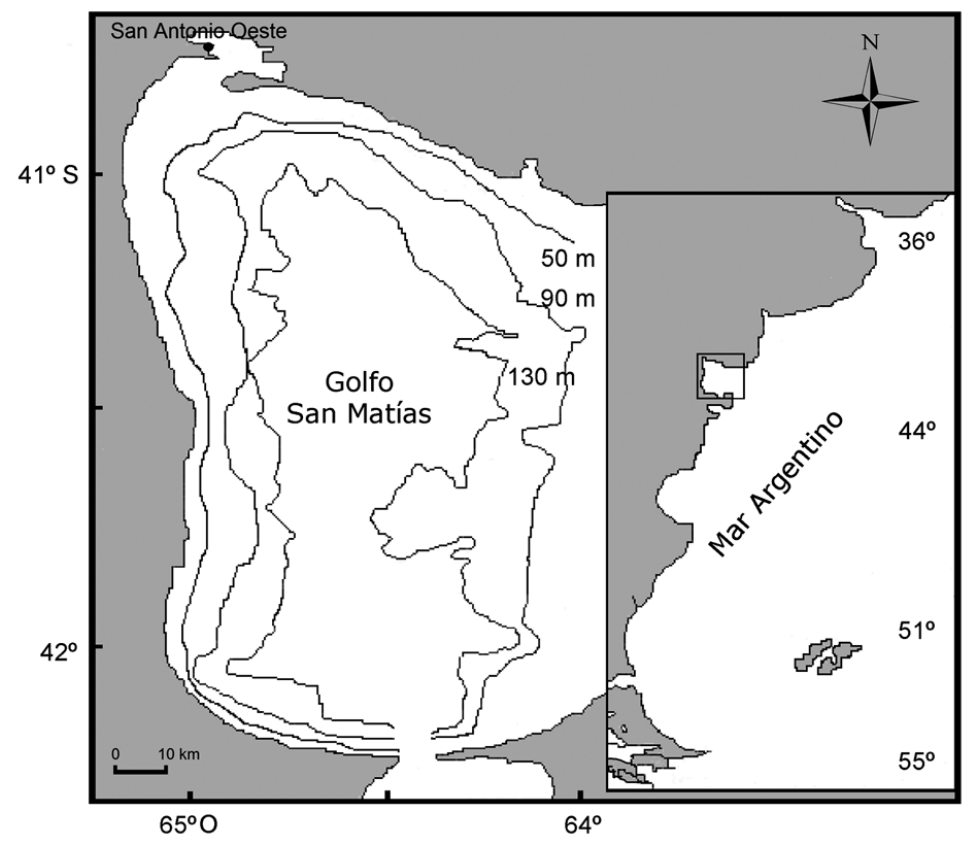

Figura 1. Ubicación geográfica del área de estudio / Geographical localization of the study area

${ }^{1}$ Millán D. 2011. Anuario de estadísticas pesqueras de la Provincia de Río Negro. Departamento Policía de Pesca, Dirección de Pesca, Reporte 2011, San Antonio Oeste, Rio Negro, Argentina 
Conocer los parámetros de historia de vida de la especie permite realizar evaluaciones de stock y la implementación de medidas de conservación y manejo para la explotación sustentable. En Argentina y más específicamente en el GSM, se conocen las características biológicas del pez gallo en cuanto a su distribución, alimentación y reproducción durante la década del 90's (Di Giácomo 1990, 1992; Di Giácomo \& Perier 1994, 1996; Di Giácomo et al. 1994). En cuanto al crecimiento, no existen antecedentes para la especie en el Atlántico sudoccidental. Los estudios de crecimiento para el pez gallo fueron realizados en el Pacífico sur mediante el análisis de estructura de tallas (Alarcón et al. 2011). A su vez se han realizado estudios de edad y crecimiento para otras especies del mismo género (C. milii y C. capensis) mediante lectura de espinas (Sullivan 1977, Freer \& Griffiths 1993, Braccini et al. 2008) y análisis de estructuras de tallas (Francis 1997). Estas estimaciones son de vital importancia ya que son los parámetros de entrada de los modelos demográficos que permiten evaluar la vulnerabilidad de este grupo de especies a diferentes escenarios de explotación pesquera.

El objetivo de este estudio fue estimar los parámetros de historia de vida del pez gallo, C. callorynchus, en el GSM $\left(41-42^{\circ} \mathrm{S}\right.$ y $\left.64-65^{\circ} \mathrm{O}\right)$, Argentina. Esta información permitirá evaluar la vulnerabilidad de la especie a la explotación pesquera y la implementación de medidas de manejo y conservación para la explotación sustentable en la región del Atlántico sudoccidental.

\section{Materiales y MÉTODOS}

\section{FUENTE dE DATOS Y ESTRUCTURA DE TALLAS}

Los ejemplares de Callorhinchus callorynchus fueron obtenidos a partir de muestreos mensuales de los desembarques comerciales y muestreos a bordo de la flota realizados desde septiembre 2011 hasta noviembre 2012. La flota pesquera opera dentro del GSM en un rango de profundidades de 50 a $190 \mathrm{~m}$. De cada individuo se registró el sexo, la talla y el estado de madurez. La talla fue medida como el largo al centímetro inferior desde el apéndice rostral hasta el comienzo del lóbulo superior de la aleta caudal (LCLS).

Se utilizó un test de $\chi^{2}$ para evaluar si la proporción de sexos difiere de la relación 1:1, considerando diferencias significativas con un $P<0,05$. Para analizar si existen diferencias en la talla media entre sexos se realizó el t-test de Welch aplicando el logaritmo al LCLS y evaluando la hipótesis nula de igualdad entre medias (Crawley 2007).
Al ser una especie capturada como by-catch, el número de individuos obtenidos mensualmente no siempre fue suficiente para la detección de las modas a partir de la estructura de tallas, por lo tanto para el análisis de crecimiento las muestras mensuales fueron agrupadas en histogramas de frecuencia de tallas trimestrales separadas por sexo con intervalos de clase de $1 \mathrm{~cm}$.

En las capturas comerciales no estuvieron representadas las modas de las tallas más chicas (LCLS $<30 \mathrm{~cm}$ ) correspondiente a las primeras clases de edad dado que la flota opera a profundidades mayores a los $50 \mathrm{~m}$ y existe segregación espacial de tallas según la batimetría (Di Giácomo 1992). Por lo tanto, para el análisis de crecimiento se incluyeron las estructuras de tallas obtenidas en las campañas de investigación de recursos demersales realizadas en el GSM durante 2005, 2006 y 2007 (REDE 0506-07). En estas muestras el rango de tallas fue mayor y estuvieron representados los ejemplares neonatos y de las primeras clases de edad (LCLS $\sim$ entre $10-30 \mathrm{~cm}$ ), dado que los lances de las campañas de investigación cubren las zonas de menor profundidad del golfo (Estalles et al. 2011) que son descriptas como áreas de cría y reclutamiento de la especie y donde no pesca la flota comercial (Di Giácomo 1992).

\section{MADUREZ}

El estado de madurez se determinó siguiendo los criterios de siguiendo a Di Giácomo \& Perier (1994). Los machos fueron clasificados según el largo y el grado de calcificación de los claspers, siendo los machos maduros los que presentaron los claspers completamente calcificados que exceden el margen posterior de la aleta pélvica. En las hembras la clasificación se basó en el tamaño y coloración de los ovocitos. Las hembras maduras estuvieron caracterizadas por presentar ovocitos amarillos de tamaño mayor a $10 \mathrm{~mm}$ de diámetro. La información del estado de madurez se utilizó en forma de datos binomiales (inmaduros $=0 \mathrm{y}$ maduros $=1$ ) los cuales fueron ajustados mediante un modelo logístico: $\mathrm{Y}=[1+$ $\exp \{-(\mathrm{a}+\mathrm{bX})\}]^{-1}$, donde $\mathrm{Y}$ es el estado de madurez, $\mathrm{X}$ es el LCLS (cm), y $a$ y $b$ son los parámetros del modelo. Se usó un modelo lineal generalizado (GLM) con distribución binomial y función de enlace logit para estimar los parámetros y la talla donde el 50\% de los individuos están maduros $\left(\operatorname{LCLS}_{50 \%}\right)$, dado por '- $a / b$ ' (Roa et al. 1999). Todos los análisis estadísticos se realizaron con el software R (R CoreTeam 2012) utilizando el paquete MASS de Venables \& Ripley (2002). 


\section{ANÁLISIS DEL CRECIMIENTo}

Se realizó un análisis de descomposición de la mezcla de distribuciones normales que están presentes en los datos de frecuencia de tallas trimestrales de machos y hembras de $C$. callorynchus. Para el análisis de mezcla o mixtura (MIX), se utilizó el programa 'mixdist' (MacDonald \& Pitcher 1979, MacDonald \& Green 1988, Du 2002) para el software R v. 2.15.1 (R Core Team 2012). El programa permite separar las clases de edad de los datos de frecuencia de tallas. Se utilizó el algoritmo quasi-newton de convergencia y se asumió que cada clase de edad tiene una función de densidad de probabilidad normal. El número de clases de edad fue determinado por inspección visual del histograma de frecuencia, y el análisis se realizó sin restringir los parámetros en una primera etapa, para posteriormente restringir en algunos casos ya sea la desviación estándar o la longitud de los grupos que no permitían convergencia en la estimación.

Una vez que todas las clases de edad con sus longitudes medias fueron separadas de cada distribución de frecuencia de tallas, se identificaron grupos de edad mediante el método de análisis de Agrupación de Cohortes (ACOH) (Roa 1993, Roa \& Ernst 1996). En el ACOH, se ordenan las longitudes medias de los grupos identificados en orden ascendentes y luego se agrupan en clases de edad relativa según saltos significativos con el método de Roa \& Ernst (1996). La ventaja de este método es que permite sumar al análisis de crecimiento muestras de estructuras de tallas de diferentes años (Roa 1993, Roa \& Ernst 1996), por lo que se incluyeron las estructuras de tallas obtenidas en las campañas de investigación REDE 05-06-07. La inclusión de estas muestras en el análisis permitió identificar las modas de las tallas más chicas (LCLS 15-30 cm, primeros grupos de edad) que no están representadas en las capturas comerciales.

El método $\mathrm{ACOH}$ tiene 2 supuestos, el primero es que individuos de las clases de edad mayores tienden a tener mayor longitud media, porque hay una función de crecimiento que regula el movimiento de las clases de edad menores a mayores. El segundo supuesto es que para cada año calendario, existe solo uno y no menos de un período de reclutamiento que genera una cohorte (clase anual). A partir de estos supuestos se realizó el siguiente procedimiento para clasificar el set de longitudes medias en clases de edad relativa: (1) Las longitudes medias identificadas se ordenaron en secuencia ascendente; (2) luego se agrupan en clases de edad relativa identificando longitudes medias como posibles límites entre las clases de edad sin incluir en una misma clase de edad 2 veces el mismo muestreo mensual o campaña de investigación; (3) por último si esta agrupación deja afuera alguna longitud media, la misma se agrupa dentro de una clase de edad donde no esté presente ese mes de muestreo considerando minimizar la alteración de la secuencia inicial. La agrupación del paso (2) se realizó comenzando de la longitud media menor hasta la mayor (forward) (Roa \& Ernst 1996).

Finalmente, una vez que se asignó la edad relativa a cada longitud media, el set de datos talla-edad se ajustó a 4 modelos de crecimiento utilizados frecuentemente en condrictios (Cailliet et al. 2006, Braccini et al. 2007). El primero fue el modelo original de von Bertalanffy (von Bertalanffy 1938) modificado con la talla de nacimiento $\mathrm{L}_{0}$ (VBGF) en vez de la edad a la talla cero $\left(\mathrm{t}_{0}\right)$, siguiendo la recomendación de Cailliet et al. (2006):

$$
\mathrm{LT}(\mathrm{t})=\mathrm{L}_{\infty}-\left(\mathrm{L}_{\infty}-\mathrm{L}_{0}\right) \mathrm{e}^{-\mathrm{kt}}
$$

donde, LT(t) es el LCLS en función del tiempo t (años), $\mathrm{L}_{\infty}$ es la talla asintótica teórica $(\mathrm{cm}), \mathrm{L}_{0}$ es la talla al nacimiento $(\mathrm{cm})$ y k es la constante de crecimiento (años ${ }^{-1}$ ). El segundo modelo fue una variación del modelo de von Bertalanffy donde solo los parámetros $\mathrm{k} \mathrm{y}_{\infty}$ son estimados $\left(\mathrm{VBGF}_{\mathrm{L} 0 \text { fijo }}\right)$, fijando la talla de nacimiento $\mathrm{L}_{0}$ en $10 \mathrm{~cm}$ que es el LCLS observado para los ejemplares neonatos del GSM (datos sin publicar). El tercer modelo fue la función de crecimiento de Gompertz (GGF) (Ricker 1975), modificada con la talla de nacimiento $\mathrm{L}_{0}$ (Romine et al. 2006):

$$
\mathrm{LT}(\mathrm{t})=\mathrm{L}_{0}\left(\mathrm{e}^{\mathrm{G}(1-\mathrm{e}(-\mathrm{k} t)}\right)
$$

donde, $\mathrm{G}=\ln \left(\mathrm{L}_{\infty} / \mathrm{L}_{\mathbf{0}}\right)$. El cuarto modelo fue el de Gompertz modificado con 2 parámetros $\left(\mathrm{GGF}_{\mathrm{L} 0 \text { fijo }}\right)$, fijando la talla nacimiento $\mathrm{L}_{0}$ en $10 \mathrm{~cm}$.

El ajuste se realizó por cuadrados mínimos no lineales (nls) con el algoritmo Gauss-Newton del software $\mathrm{R}$ v.2.15.1 (R Core Team 2012) y para cada modelo se estimaron sus parámetros y el error estándar (EE). El ajuste de los modelos se evaluó según un criterio estadístico basado en el Criterio de Información de Akaike ajustado para muestras pequeñas (AICc) (Burnham \& Anderson 2002) y en el criterio biológico basado en que los parámetros estimados sean biológicamente posibles (Cailliet et al. 2006). El valor de Akaike corregido para muestras pequeñas se estimó como $\mathrm{AICc}=\mathrm{AIC}+$ $(2 k(k+1)) /(n-k-1)$, donde para cuadrados mínimos $\mathrm{AIC}=n$ $\log \left(\sigma^{2}\right)+2 k, \sigma^{2}=\mathrm{SCR} / n, \mathrm{SCR}$ es la suma de cuadrados residual, $n$ el número de observaciones y $k$ es el total de parámetros estimados en el modelo incluyendo $\sigma^{2}$; siendo 
el modelo de mejor ajuste el que tiene el menor valor de $\mathrm{AICc}\left(\mathrm{AICc}_{\min }\right)$. Las diferencias en el valor de $\mathrm{AICc}$ fueron calculadas como $\Delta_{\mathrm{i}}=\mathrm{AICc}_{\mathrm{i}}-\mathrm{AICc}_{\text {min }}$, donde $\mathrm{AICc}_{\mathrm{i}}$ es el valor de Akaike del modelo $i$-ésimo. Los valores de $\Delta_{i}$ fueron usados para realizar un ranking de cada modelo en relación al modelo de mejor ajuste. Modelos con $\Delta_{\mathrm{i}}$ de 0 2 tienen un buen sustento de los datos ajustados, mientras que modelos con $\Delta_{\mathrm{i}}$ entre 4-7 tienen considerablemente menor sustento. Modelos con $\Delta_{i}>10$ prácticamente no tienen ningún apoyo estadístico (Burnham \& Anderson 2002). Los pesos de Akaike ( $\mathrm{w}_{\mathrm{i}}$ ) fueron calculados para ver cuál es el modelo de mejor ajuste entre todos los modelos candidatos (Burnham \& Anderson 2002).

\section{MORTALIDAD NATURAL Y LONGEVIDAD}

Las máximas estimaciones de edades proveen una aproximación inicial de la longevidad, sin embargo estos valores pueden subestimarla si la población ha estado bajo explotación comercial. Por lo tanto se estimó la longevidad teórica $\left(\mathrm{E}_{\max }\right)$ como la edad en el cual los ejemplares alcanzan el 95\% de la talla asintótica $\mathrm{L}_{\infty}$ (Taylor 1958, Natanson et al. 2006). A su vez, la tasa de mortalidad natural (M) fue estimada a partir de 4 modelos empíricos utilizados frecuentemente en condrictios (Simpfendorfer et al. 2005, Alarcon et al. 2011):

1) Pauly (1980): este autor encontró una relación entre la tasa de mortalidad natural, el coeficiente de crecimiento (K) y longitud asintótica $\left(\mathrm{L}_{\infty}\right)$ del modelo de von Bertalanffy, y la temperatura anual promedio del hábitat (T), utilizando datos de 175 stocks de peces. El modelo de regresión es:

$\log _{10} \mathrm{M}=-0,0066-0,27 \log _{10} \mathrm{~L}_{\infty}+0,6543 \log _{10} \mathrm{~K}+$ $0,4634 \log _{10} \mathrm{~T}$

Se utilizaron los parámetros $\mathrm{L}_{\infty}$ y $\mathrm{K}$ del modelo $\mathrm{GGF}_{\mathrm{L} 0 \text { fijo }}$ y la $\mathrm{T}$ promedio de $10,85^{\circ} \mathrm{C}$ ya que el rango de temperaturas de fondo registrado en el GSM es entre 10,4 y $11,3^{\circ} \mathrm{C}$ (Perier \& Di Giácomo 2002).

2) Hoenig (1983): este autor encontró una relación empírica entre la tasa de mortalidad total $(Z)$ y la edad máxima observada $\left(t_{\max }\right)$ de varias especies marinas. Muchos de los datos pertenecen a stocks no explotados o levemente explotados, de tal manera que $Z \sim M$. El modelo de regresión es:

$$
\log _{\mathrm{e}} Z=1,44-0,982 \log _{\mathrm{e}} \mathrm{E}_{\max }
$$

donde, $\mathrm{Z}$ es la tasa de mortalidad natural $\mathrm{Z}(\sim \mathrm{M}), \mathrm{y}_{\max }$ es la máxima edad observada para hembras y machos. Sin embargo, debido a la falta de una estimación directa de la edad en este estudio, fue utilizada como $\mathrm{E}_{\max }$ la longevidad estimada.

3) Rickhter \& Efanov (1976): Estos autores proponen la siguiente relación:

$$
\mathrm{M}=\left(1,521 / \mathrm{E}_{50 \%}^{0,72}\right)-0,155
$$

donde $\mathrm{E}_{50 \%}$ es la edad de madurez, estimada usando el LT50\% y los parámetros del modelo de $\mathrm{GGF}_{\mathrm{L} 0 \text { fijo }}$.

4) Jensen (1996): este autor reanalizó los datos y la relación de Pauly (1980) y propuso 3 fórmulas más simples entre los parámetros de historia de vida y la mortalidad natural:

$$
\begin{aligned}
& M=1,65 / \mathrm{E}_{50 \%} \\
& M=1,5 \mathrm{~K} \\
& \mathrm{M}=1,6 \mathrm{~K}
\end{aligned}
$$

\section{Resultados}

\section{Muestras ObTenidas Y eSTRUCTURa de TallaS}

El rango de tallas obtenido a partir de los muestreos de desembarques de las capturas comerciales entre octubre 2011-septiembre 2012 estuvo comprendido entre 22 y 58 $\mathrm{cm}(\mathrm{n}=2126)$ para los machos y entre 25 y $76 \mathrm{~cm}(\mathrm{n}=1571)$ para las hembras (Fig. 2). La proporción sexual para el total de los muestreos difirió significativamente de la relación 1:1 esperada $\left(\chi_{(0,05,1)}^{2}=83,32, P<0,05\right)$, con mayor número de ejemplares machos que hembras (relación 1:0,7). Al analizar la proporción sexual por trimestres, se observó el mismo patrón en el $1^{\mathrm{er}}$ y $4^{\text {to }}$ trimestre $\left(1^{\mathrm{er}}\right.$ trimestre: $\chi_{(0,05,1)}^{2}=123,23, P<0,05$ y $4^{\text {to }}$ trimestre: $\chi_{(0,05,1)}^{2}=$ $37,2, P<0,05)$, en cambio en el $2^{\text {do }}$ y $3^{\text {er }}$ trimestre del año no se observaron diferencias significativas de la relación 1:1 $\left(2^{\text {do }}\right.$ trimestre: $\chi_{(0,05,1)}^{2}=2,46, P>0,12$ y $3^{\text {er }}$ trimestre: $\left.\chi_{(0,05,1)}^{2}=3,5, P>0,05\right)$.

Existieron diferencias significativas entre la talla media de machos y hembras en la región de $\operatorname{GSM}\left(t_{(0,05,2396)}=\right.$ $-22,6303, P<0,001)$, siendo la talla promedio de los machos $\left(\operatorname{LCLS}_{\text {medio }}=43,7 \mathrm{~cm}\right.$; desv. est. $\left.\pm 4,4\right)$ inferior a la talla de las hembras ( $\operatorname{LCLS}_{\text {medio }}=49,6 \mathrm{~cm}$; desv. est. $\pm 8,3$ ). El rango de tallas obtenido a partir de las 3 campañas de investigación fue entre 8 a $59 \mathrm{~cm}$ LCLS para los machos $(\mathrm{n}=1005)$ y entre 14 a $69 \mathrm{~cm}$ de LCLS para las hembras $(\mathrm{n}=$ 1132) (Fig. 2). 
a) Capturas comerciales
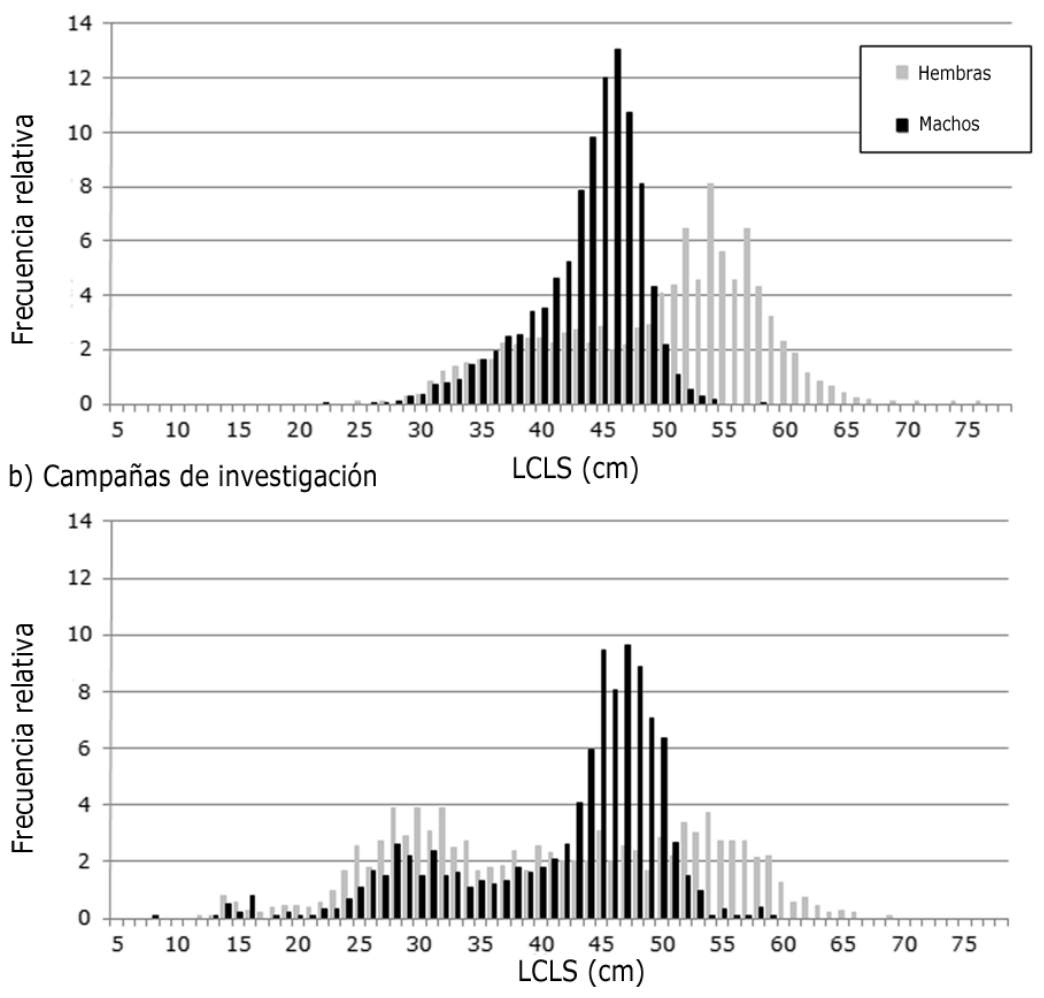

Figura 2. Estructura de tallas del pez gallo en el Golfo San Matías. a) Muestra de las capturas comerciales entre octubre 2011-septiembre 2012: hembras ( $n=1571)$ en gris y machos $(n=2126)$ en negro. b) M uestra obtenida a partir de las campañas de investigación REDE 2005-2006-2007; hembras $(n=1132)$ en gris y machos $(n=1005)$ en negro / Length frequency data of cockfish in the San Matías gulf. a) Commercial catch samples between October 2011-september 2012: females $(n=1571)$ in grey and males $(n=2126)$ in black. b) Samples of fishery surveys REDE 2005-2006-2007: females $(n=1132)$ in grey and males $(n=1005)$ in black

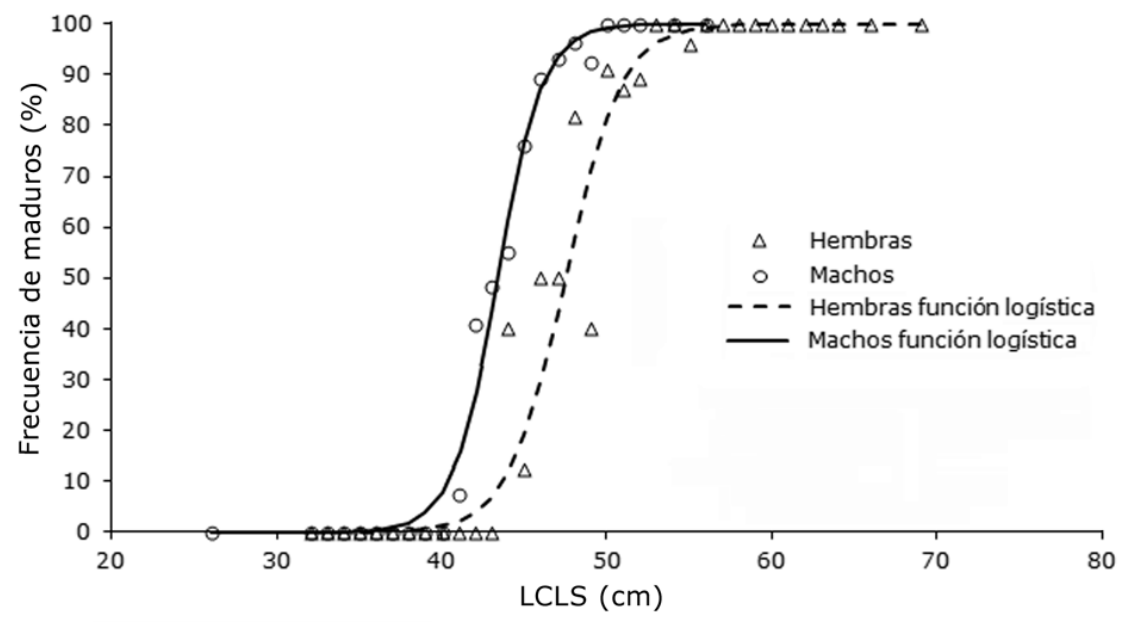

Figura 3. Proporción de hembras y machos maduros en función de la talla (LCLS) ajustados a la función logística: ajuste del modelo (línea continua y punteada); proporciones observadas de hembras y machos maduros / The proportion of mature females and males as a function of body length (LCLS) modeled with a logistic function: the model fitted (continuous line and dotted line); observed proportions of mature females and males 


\section{MADUREZ}

El $\mathrm{LM}_{50 \%}$ estimado para los machos fue de $43,39 \mathrm{~cm}$ (IC: 43,05-43,74, n=600). Los parámetros del MLG fueron a= -31,895 (Error estándar, $\mathrm{s}_{\overline{\mathrm{x}}}: 2,807$ ) y b= 0,735 (Error estándar, $\left.\mathrm{s}_{\overline{\mathrm{x}}}: 0,0635\right)$, con un $96 \%$ de la devianza explicada por el modelo. El $\mathrm{LM}_{50 \%}$ estimado para las hembras fue de 47,48 cm (IC: 46,67 - 48,28, n= 398). Los parámetros del MLG fueron $\mathrm{a}=-27,826$ (Error estándar, $\mathrm{s}_{\overline{\mathrm{x}}}: 3,439$ ) y $\mathrm{b}=0,586$
(Error estándar, $\mathrm{s}_{\overline{\mathrm{x}}}$ : 0,0699), con un 97\% de la devianza explicada (Fig. 3).

\section{Análisis de CRECIMIENTo}

Los datos de frecuencia de tallas utilizados para el análisis del crecimiento y las longitudes medias detectadas con MIX se presentan en las Fig. 4 y 5. Los histogramas de a) Machos
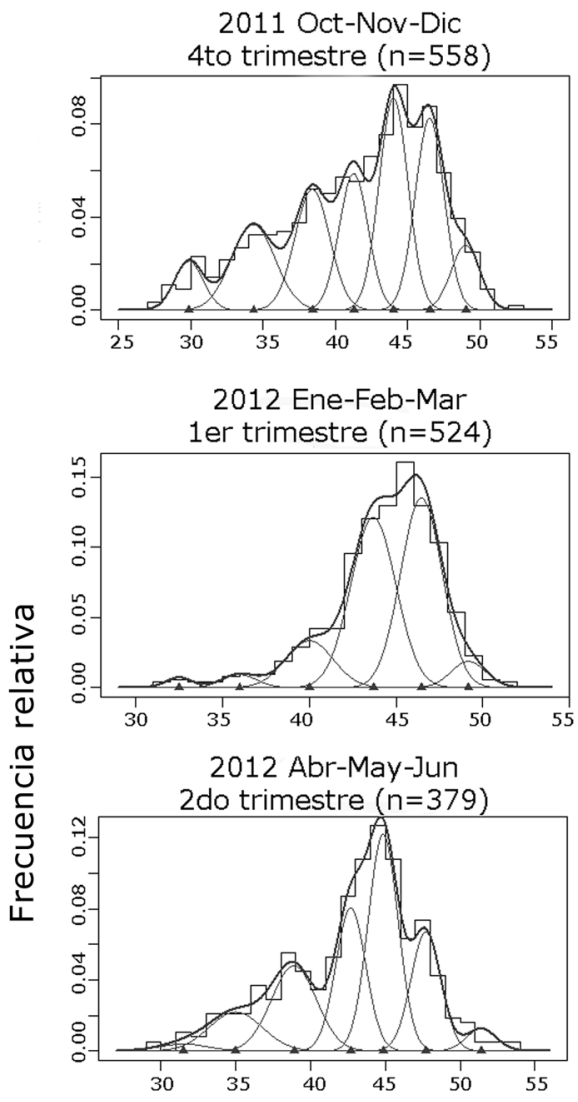

2012 Jul-Ago-Sep 3er trimestre $(n=305)$

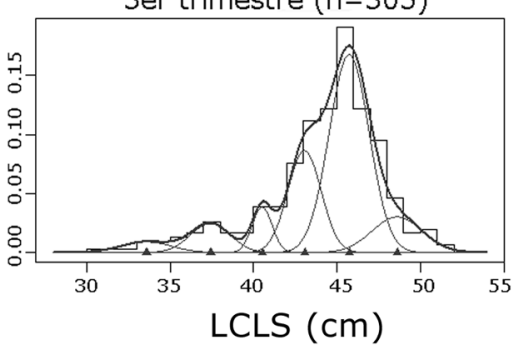

b) Hembras

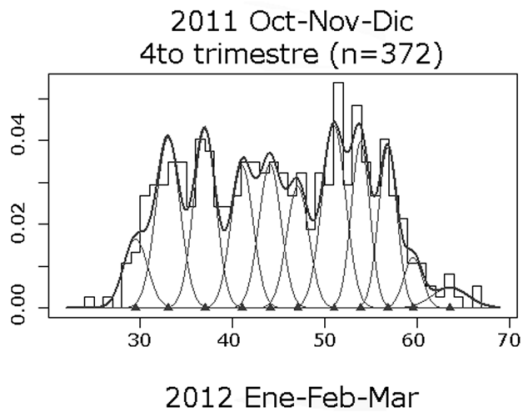

1er trimestre $(\mathrm{n}=221)$

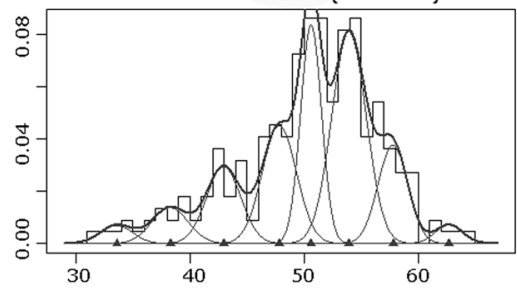

2012 Abr-May-Jun

2do trimestre $(n=337)$

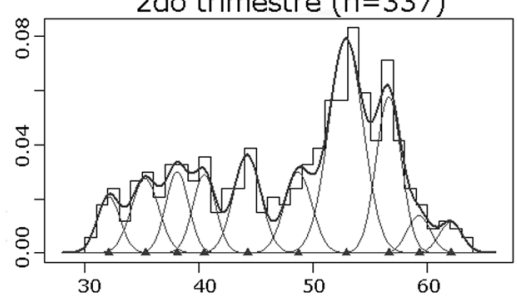

2012 Jul-Ago-Sep

3er trimestre $(n=353)$

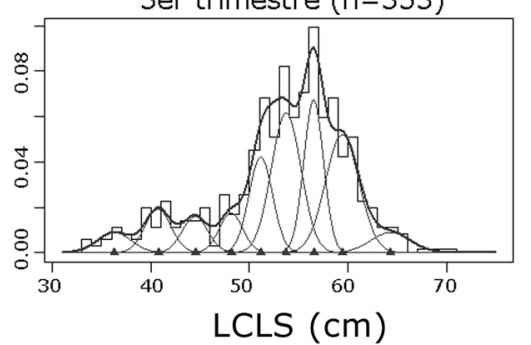

Figura 4. Set de datos de frecuencia de tallas trimestrales (histogramas) de las capturas comerciales. Las líneas internas representan la distribución normal de las tallas medias identificadas para cada clase de edad (triángulos), y la línea externa representa la frecuencia de tallas estimada por el programa MIX / Quarters length-frequency data set (histograms) of commercial catch. The thin lines inside represent the normal distribution of identified length at a given age class (triangles), and the outer thick line represent the estimated length-frequency by MIX method 
a) Machos

REDE05 - 2005 diciembre $(n=156)$

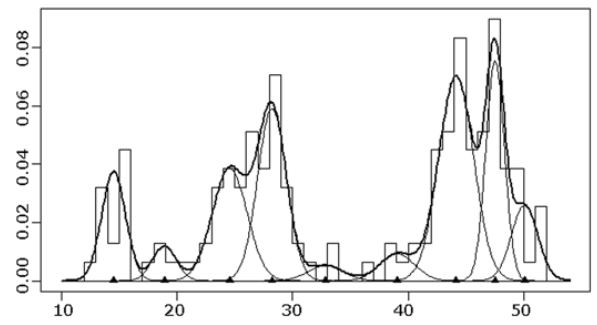

REDE06 - 2006 noviembre $(n=401)$

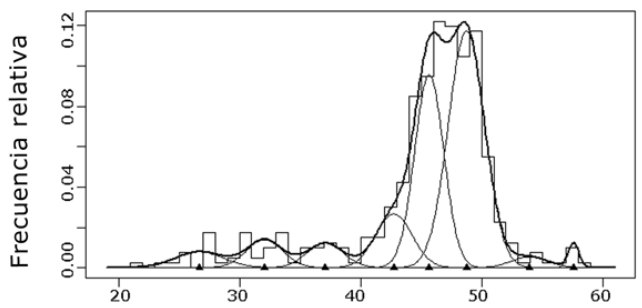

REDE07 - 2007 octubre $(n=446)$

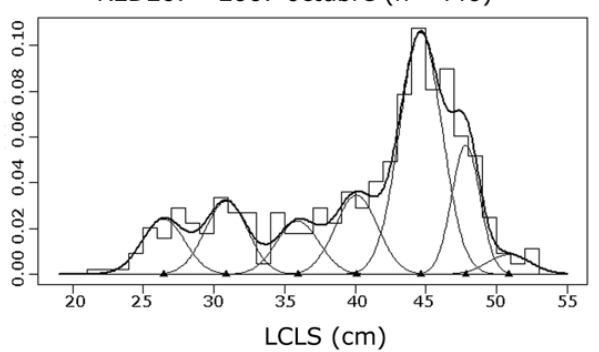

b) Hembras
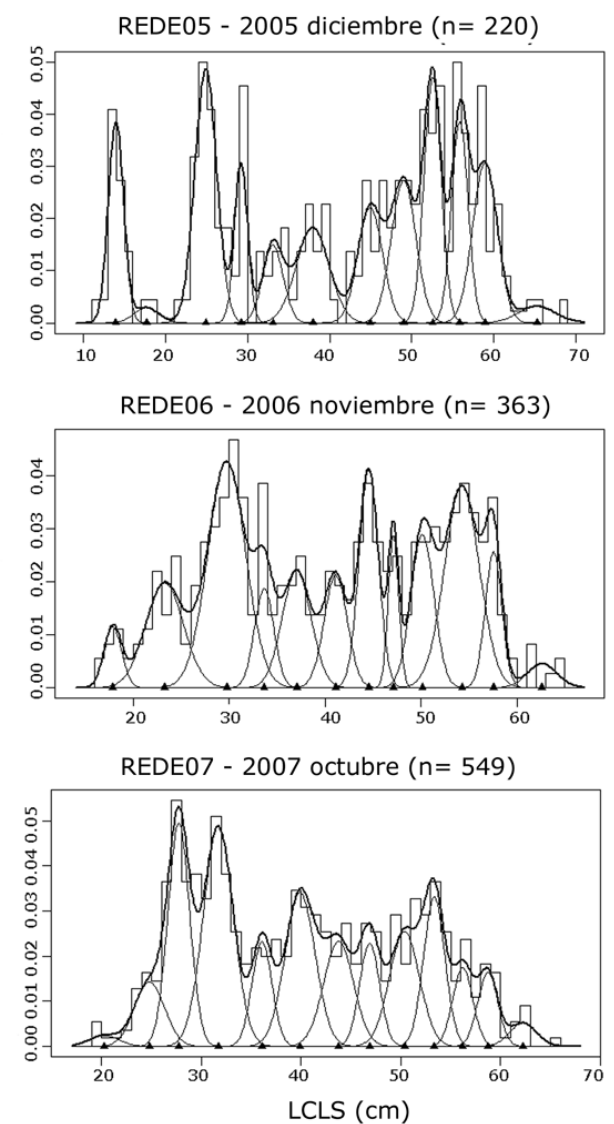

Figura 5. Set de datos de frecuencia de tallas del pez gallo obtenidas de las campañas de investigación REDE 05-06-07 (histogramas). Las líneas internas representan la distribución normal de las tallas medias identificadas para cada clase de edad (triángulos), y la línea externa representa la frecuencia de tallas estimada por el programa MIX / Quarters length-frequency data set (histograms) of the fisheries surveys REDE 05-0607. The thin lines inside represent the normal distribution of identified length at a given age class (triangles), and the outer thick line represent the estimated length-frequency by MIX method

frecuencia de tallas de machos y hembras muestran una forma polimodal, que implica la presencia de varios grupos en la mezcla de tallas. En las muestras de frecuencia de tallas trimestrales del período 2011-2012 el número de longitudes medias detectadas para los machos varió entre 6 y 7 con valores de longitud media entre 29,89 y 51,42 $\mathrm{cm}$, identificándose en total para todo el set de datos 26 longitudes medias. En cambio, para las hembras el número de longitudes medias determinado con MIX en cada frecuencia de tallas mensual varió entre 8 y 11 , con valores entre 29,54 y $63,61 \mathrm{~cm}$, identificándose en total 38 longitudes medias en todo el set de datos (Fig. 4). Cuando se incluyeron los datos de las campañas de investigación, el número de longitudes medias identificado con MIX en cada estructura de tallas fue mayor que en los muestreos de las capturas comerciales. Para los machos se identificaron entre 7 y 9 longitudes medias con valores entre 14,53 y $57,6 \mathrm{~cm}$; y para las hembras se identificaron entre 12 y 13 longitudes medias con valores entre 13,93 y 65,24 cm (Fig. 5).

Mediante el ACOH se identificaron para los machos 11 clases de edad y para las hembras 16 clases de edad, lo que muestra que las hembras tienen ejemplares de mayor tamaño y edades respecto a los machos (Tabla 1 y Fig. 6). En la Tabla 2 se presentan los parámetros estimados para cada modelo evaluado. Para las hembras el criterio estadístico indica que los modelos de VBGF y $\mathrm{VBGF}_{\mathrm{L} 0 \text { fijo }}$ son los más adecuados dado que presentaron el mismo valor de AICc $(15,39)$ y w $_{\mathrm{i}}(0,5)$. En el caso de los machos, el modelo de $\mathrm{VBGF}_{\mathrm{L} 0 \text { fijo }}$ fue el más adecuado ya que presentó el menor valor de AICc $(14,31)$ y mayor $\mathrm{w}_{\mathrm{i}}(0,4)$. 
Tabla 1. Clases de edad relativa identificadas mediante $\mathrm{ACOH}$, talla media estimada (LCLS), desviación estándar (DE) y número de muestras donde fue identificada la clase de edad (N). Período septiembre de 2011-octubre de 2012 y campañas de investigación REDE 05-06-07 / Relative age classes identified with $\mathrm{ACOH}$, estimated mean length (LCLS), standard deviation (DE) and number of samples where were identified the age classes (N). Samples period September 2011 to October 2012 and fishery surveys REDE 05-06-07

\begin{tabular}{|c|c|c|c|c|c|c|}
\hline \multirow{2}{*}{$\begin{array}{l}\text { Clases } \\
\text { de edad } \\
\text { relativa }\end{array}$} & \multicolumn{3}{|c|}{ Machos } & \multicolumn{3}{|c|}{ Hembras } \\
\hline & $\begin{array}{l}\text { LCLS } \\
(\mathrm{cm})\end{array}$ & $\begin{array}{l}\mathrm{DE} \\
(\mathrm{cm})\end{array}$ & $\mathrm{N}$ & $\begin{array}{c}\text { LCLS } \\
(\mathrm{cm})\end{array}$ & $\begin{array}{l}\mathrm{DE} \\
(\mathrm{cm})\end{array}$ & $\mathrm{N}$ \\
\hline 1 & 14,53 & - & 1 & 13,93 & - & 1 \\
\hline 2 & 18,91 & - & 1 & 18,60 & 1,45 & 3 \\
\hline 3 & 25,90 & 1,16 & 3 & 24,33 & 0,90 & 3 \\
\hline 4 & 30,85 & 1,58 & 6 & 29,06 & 0,91 & 4 \\
\hline 5 & 34,98 & 1,48 & 7 & 32,87 & 0,80 & 6 \\
\hline 6 & 38,99 & 1,01 & 6 & 36,35 & 0,73 & 5 \\
\hline 7 & 41,82 & 1,09 & 4 & 38,10 & 0,18 & 3 \\
\hline 8 & 44,08 & 0,65 & 6 & 40,67 & 0,49 & 5 \\
\hline
\end{tabular}

\begin{tabular}{|c|c|c|c|c|c|c|}
\hline \multirow{2}{*}{$\begin{array}{l}\text { Clases } \\
\text { de edad } \\
\text { relativa }\end{array}$} & \multicolumn{3}{|c|}{ Machos } & \multicolumn{3}{|c|}{ Hembras } \\
\hline & $\begin{array}{c}\text { LCLS } \\
(\mathrm{cm})\end{array}$ & $\begin{array}{c}\mathrm{DE} \\
(\mathrm{cm})\end{array}$ & $\mathrm{N}$ & $\begin{array}{c}\text { LCLS } \\
(\mathrm{cm})\end{array}$ & $\begin{array}{c}\mathrm{DE} \\
(\mathrm{cm})\end{array}$ & $\mathrm{N}$ \\
\hline 9 & 46,79 & 0,91 & 7 & 44,16 & 0,64 & 7 \\
\hline 10 & 49,71 & 1,11 & 7 & 47,84 & 0,83 & 7 \\
\hline 11 & 53,96 & - & 1 & 50,67 & 0,43 & 5 \\
\hline 12 & & & & 53,53 & 0,63 & 7 \\
\hline 13 & & & & 56,78 & 0,70 & 7 \\
\hline 14 & & & & 59,20 & 0,36 & 5 \\
\hline 15 & & & & 62,42 & 0,30 & 4 \\
\hline $16+$ & & & & 64,39 & 0,82 & 3 \\
\hline
\end{tabular}

Tabla 2. Parámetros de crecimiento para machos y hembras de C. callorynchus, estimados con los datos LCLS-edad relativa. VBGF= modelo de

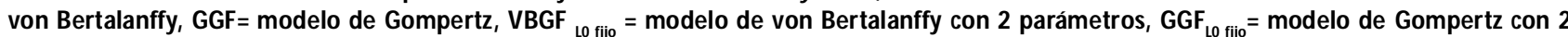
parámetros. $L_{\infty}$ es la talla asintótica $(\mathrm{cm}) ; L_{0}$ es la talla de nacimiento $(\mathrm{cm})$, $\mathrm{k}$ es la constante de crecimiento (años $\left.{ }^{-1}\right)$; AICc= valor del criterio de información de Akaike; $\Delta_{\mathrm{i}}$ es la diferencia del valor AIC entre el modelos; $\mathrm{w}_{\mathrm{i}}$ es el peso de Akaike y $\mathrm{E}_{\max }$ es la longevidad teórica máxima (años). Entre paréntesis se informa el error estándar (EE) de cada parámetro / Parameters for each growth model for males and females of $\mathrm{C}$. callorynchus estimated with the length LCLS-relative ages data. VBGF= von Bertalanffy model, GGF= Gompertz model, VBGF Lo fijo $=$ von Bertalanffy model with two parameters, $G_{G F} F_{L \text { fijo }}=$ Gompertz model with two parameters. $L_{\infty}$ is the theoretical asymptotic length $(\mathrm{cm}) ; L_{0}$ is the size at birth (cm), $\mathrm{K}$ is the growth coefficient (year ${ }^{-1}$ ); AICc= is the modified Akaike's Information Criterion; $\Delta_{\text {i }}$ is the AICc differences; $\mathrm{w}_{\mathrm{i}}$ is the Akaike weights and $\mathrm{E}_{\max }$ is the maximum theoretical longevity (years). In brackets there is the standard error (SE) for each estimated parameter

\begin{tabular}{|c|c|c|c|c|c|c|c|c|}
\hline Sexo & Modelo & $\mathrm{K}$ & $\mathrm{L}_{\infty}$ & $\mathrm{L}_{0}$ & AICc & $\Delta_{\mathrm{i}}$ & $\mathrm{w}_{\mathrm{i}}$ & $\mathrm{E}_{\max }$ \\
\hline \multirow[t]{8}{*}{ Machos } & VBGF & 0,125 & 66,37 & 7,37 & 15,11 & 0,8 & 0,27 & 23,0 \\
\hline & & $(0,015)$ & $(3,27)$ & $(1,40)$ & & & & \\
\hline & VBGF $_{0}$ fijo & 0,100 & 72,84 & 10 & 14,31 & 0 & 0,40 & 28,5 \\
\hline & & $(0,007)$ & $(3,01)$ & $(-)$ & & & & \\
\hline & GGF & 0,238 & 57,44 & 10,99 & 17,86 & 3,55 & 0,07 & 14,6 \\
\hline & & $(0,019)$ & $(1,68)$ & $(0,95)$ & & & & \\
\hline & $\mathrm{GGF}_{0}$ fijo & 0,257 & 56,11 & 10 & 15,1 & 0,79 & 0,27 & 13,7 \\
\hline & & $(0,007)$ & $(0,89)$ & $(-)$ & & & & \\
\hline \multirow[t]{8}{*}{ Hembras } & VBGF & 0,039 & 125,04 & 11,62 & 15,39 & 0 & 0,50 & 74,3 \\
\hline & & $(0,005)$ & $(11,02)$ & $(0,69)$ & & & & \\
\hline & VBGF $_{0}$ fijo & 0,049 & 108,55 & 10 & 15,39 & 0 & 0,50 & 59,2 \\
\hline & & $(0,003)$ & $(4,26)$ & $(-)$ & & & & \\
\hline & GGF & 0.114 & 85,01 & 14,65 & 24,79 & 9,4 & 0,00 & 31,0 \\
\hline & & $(0,007)$ & $(3,19)$ & $(0,56)$ & & & & \\
\hline & $\mathrm{GGF} \mathrm{L}_{0 \text { fijo }}$ & 0,17 & 70,75 & 10 & 45,3 & 29,91 & 0,00 & 21,4 \\
\hline & & $(0,004)$ & $(1,25)$ & $(-)$ & & & & \\
\hline
\end{tabular}


a) Machos

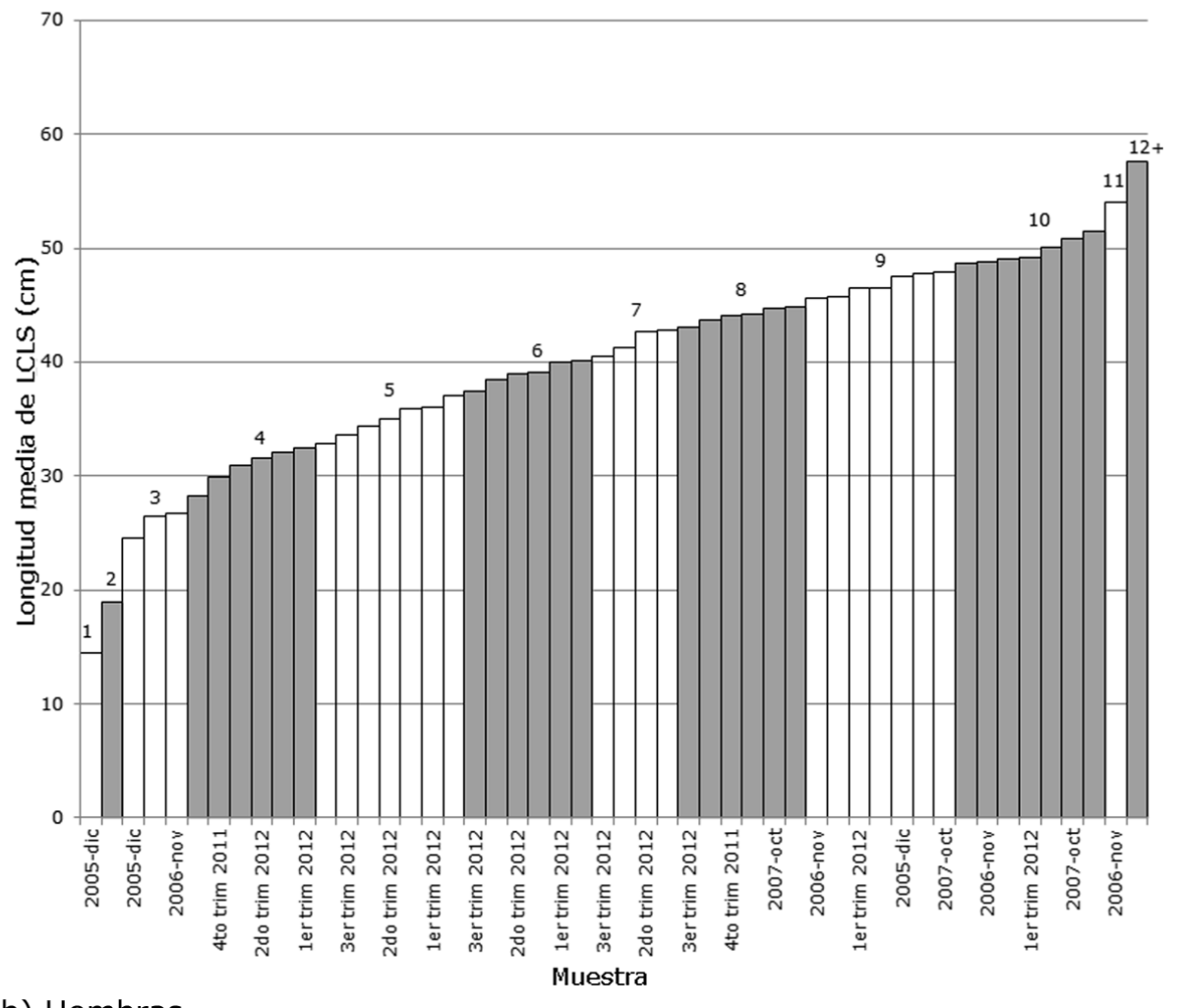

b) Hembras

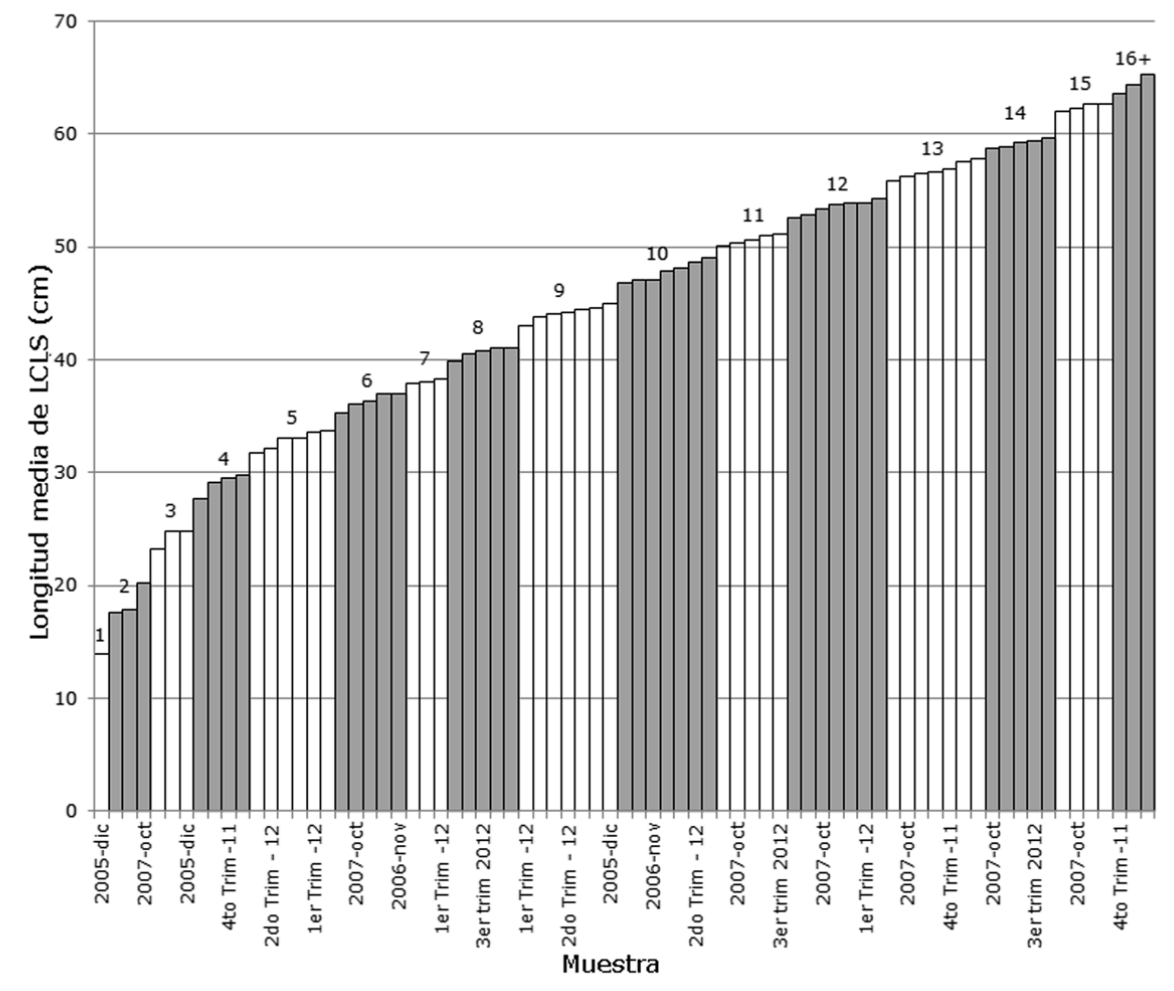

Figura. 6. Longitudes medias agrupadas en clases de edad por sexo. El número superior indica cada clase de edad agrupadas por color, mientras las barras son las longitudes medias identificadas en cada muestra trimestral / Mean lengths grouped in age classes by sex. The age classes are identified with a number and contrasting tones, while the bars corresponding to mean lengths cohorts identified within each trimester samples 
Sin embargo, estos modelos a pesar de ser los de mayor sustento estadístico, el $\mathrm{L}_{\infty}$ obtenido fue biológicamente imposible ya que en ambos sexos sobrestimó los valores de LCLS máximos registrados en el GSM. Por lo tanto considerando el criterio biológico el modelo de $\mathrm{GGF}_{\mathrm{L} 0 \text { fijo }}$ fue seleccionado como el más adecuado para ambos sexos

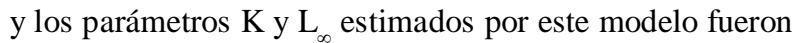
utilizados para calcular la mortalidad. En este caso los $\mathrm{L}_{\infty}$ estimados (Tabla 1) fueron similares a los valores registrados en el GSM, tanto para machos (LCLS $_{\max }=62$ cm, Di Giácomo 1992) como para hembras $\left(\right.$ LCLS $_{\max }=76$ cm, obtenido en este estudio). En la Fig. 7 se observan las curvas de crecimiento ajustadas al set de datos de talla media-clase de edad relativa obtenidos para cada sexo.

\section{LONGEVIDAD Y MORTALIDAD NATURAL}

La longevidad teórica $\left(\mathrm{E}_{\max }\right)$ estimada con los parámetros de cada modelo se muestra en la Tabla 2. Según el modelo de GGF $\mathrm{L}_{0 \text { fijo }}$ seleccionado como el más adecuado, los machos alcanzarían una $\mathrm{E}_{\max }$ de 13,7 años y las hembras de 21,4 años.

a) Machos

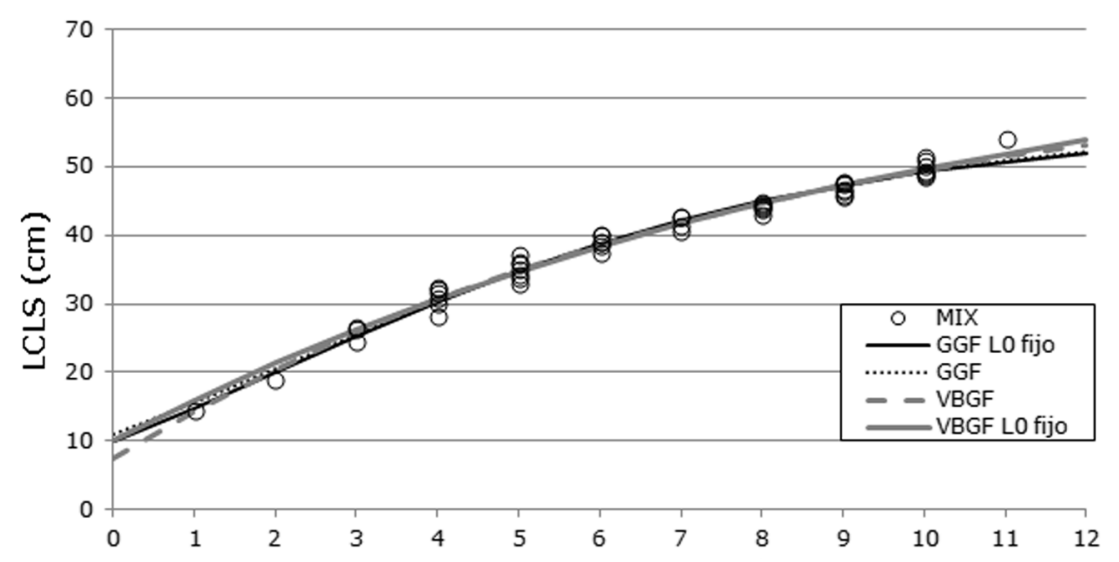

b) Hembras

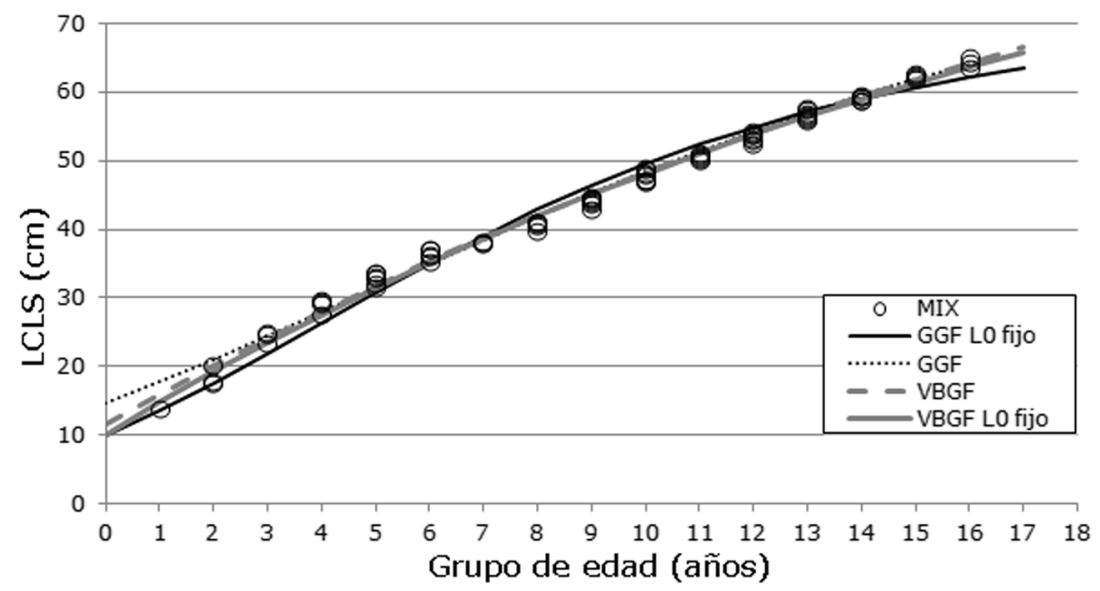

Figura 7. Ajuste de los modelos de crecimiento a los datos de LCLS media-edad relativa de las cohortes (identificadas con MIX) para cada sexo.

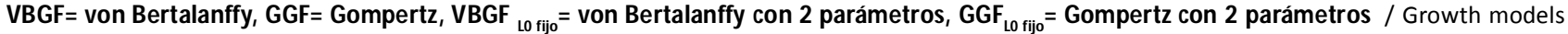
fitted to the identified length-at-relative age data of all cohorts (MIX) for each sex. VBGF= von Bertalanffy, GGF= Gompertz, VBGF $L 0$ fijo $=$ von Bertalanffy with 2 parameters, $\mathrm{GGF}_{\mathrm{L} \text { fijo }}=$ Gompertz with 2 parameters 
$\mathrm{Al}$ considerar una temperatura media anual de $10,85^{\circ} \mathrm{C}$ para el hábitat de $C$. callorynchus, el modelo de Pauly (1980) determinó una tasa de mortalidad natural igual a 0,41 año $^{-1}$ para machos y 0,30 año $^{-1}$ para hembras. Para ambos sexos los menores valores de $\mathrm{M}$ se obtuvieron con la fórmula de Rickhter \& Efanov (1976) siendo 0,28 y 0,15 año $^{-1}$ para machos y hembras respectivamente. Con la fórmula de Hoenig (1983) se obtuvo un valor intermedio respecto a las otras dos estimaciones, siendo $\mathrm{M}$ de 0,32 año ${ }^{-1}$ para machos y 0,21 año $^{-1}$ para hembras. Por último con las fórmulas propuestas por Jensen (1996) la M estimada fue de $0,41,0,38$ y 0,29 año $^{-1}$ para machos, mientras que para hembras fue de 0,27, 0,25 y 0,18 año $^{-1}$. El promedio de $\mathrm{M}$ considerando todas las estimaciones fue 0,35 año ${ }^{-1}$ para machos y 0,23 año $^{-1}$ para hembras.

\section{Discusión}

\section{Estructura de tallas}

La estructura de tallas obtenida muestra que la longitud máxima alcanzada por las hembras es mayor a la de los machos, lo que coincide con lo reportado en otros estudios para la especie (Di Giácomo 1992, Alarcón et al. 2011), siendo un patrón común en otras especies del mismo género como C. milli (Sullivan 1977, Francis 1997) y C. capensis (Freer \& Griffiths 1993).

La proporción de sexos sesgada hacia machos es frecuente en los muestreos de las capturas comerciales del GSM. En este estudio se obtuvo ese patrón para el total de los muestreos de los desembarques comerciales, coincidiendo con lo reportado por Di Giácomo (1992). Este autor observó una proporción sexual con predominancia de machos en todos los estratos de profundidad del GSM, a excepción de una zona de reclutamiento (profundidad entre 20-35 m), abundante en juveniles (LCLS 23-41 cm) donde la relación fue 1:1. Para explicar este patrón Di Giácomo (1990) plantea 2 hipótesis: migraciones estacionales de las hembras con fines reproductivos o segregación espacial de sexos. En este estudio se observó que la proporción de sexos no difirió significativamente de la relación 1:1 durante el $2^{\text {do }}$ y $3^{\text {er }}$ trimestre del año, lo que podría deberse a una modificación de la zona de pesca de la flota o a las hipótesis planteadas por Di Giácomo (1990), sin embargo estas no pudieron ser evaluadas con la información disponible en este trabajo.

Por otro lado, la diferencias entre las estructuras de tallas obtenidas en los muestreos de las capturas comerciales y en las campañas de investigación indican que la pesca estaría impactando principalmente sobre la fracción adulta de la población y confirman lo descrito por Di Giácomo (1992), en cuanto a que las zonas costeras son áreas de cría y reclutamiento de la especie. En este estudio las campañas de investigación abarcaron zonas costeras de donde se obtuvieron muestras con clases de edad y tallas de neonatos y juveniles. Proteger estas zonas de la pesca comercial mediante vedas o áreas protegidas es importante para disminuir el impacto en los ejemplares juveniles de la población, permitiendo el crecimiento hasta alcanzar la talla de madurez y el reclutamiento de las nuevas cohortes (Dulvy \& Forrest 2010).

\section{Madurez SeXual}

Di Giácomo \& Perier (1994) reportaron para el pez gallo del GSM una talla promedio de madurez de $49 \mathrm{~cm}$ y $40 \mathrm{~cm}$ de LCLS para las hembras y machos, respectivamente, que difieren levemente con los valores de $\mathrm{LM}_{50 \%}$ estimados en este estudio $(47,48 \mathrm{~cm}$ para hembras y 43,39 $\mathrm{cm}$ machos). Si bien los criterios de madurez utilizados fueron los mismos, Di Giácomo \& Perier (1994) no realizaron un ajuste al modelo logístico de los datos observados lo que podría explicar las diferencias. Para el Pacífico, Alarcón et al. (2011) reportaron un $\mathrm{LM}_{50 \%}$ de 43,7 cm para los machos, lo que coincide con el valor obtenido en el GSM ya que se encuentra dentro del intervalo de confianza estimado (43,05-43,74 cm). En cambio, en el caso de las hembras del Pacífico se obtuvo $\mathrm{LM}_{50 \%}$ de 50,2 cm, que es 2,7 cm mayor al obtenido en el GSM. Esta diferencia podrían deberse al escaso número de ejemplares $(n=40)$ utilizado por Alarcón et al. (2011). A pesar de estas leves diferencias en la talla de madurez reportadas, todos los estudios indican que existe dimorfismo sexual en la madurez del pez gallo, alcanzando las hembras la madurez a mayor talla que los machos. Este patrón es común en otras especies de holocéfalos (Freer \& Griffiths 1993, Moura et al. 2004, Barnett et al. 2009) y elasmobranquios (Cortés 2000).

\section{Crecimiento}

Los parámetros de crecimiento obtenidos en este estudio por el método Mix-ACOH para C. callorynchus del GSM, indican que existe dimorfismo sexual en el crecimiento, teniendo las hembras un crecimiento de tipo moderado $\left(0,1\right.$ años $^{-1}>\mathrm{K}<0,20$ años $\left.^{-1}\right)$ mientras que en los machos es de tipo rápido $\left(\mathrm{K}>0,2\right.$ años $\left.^{-1}\right)$. La longitud asintótica obtenida con los modelos de $\mathrm{GGF}_{\mathrm{L} 0 \text { fijo }}$, de $56,11 \mathrm{~cm}$ para los machos y de $70,75 \mathrm{~cm}$ para las hembras son razonables considerando que la longitud máxima observada en los 
Tabla 3. Estimaciones del parámetro $K$ para diferentes especies del género Callorhinchus. VBGF= modelo de crecimiento de Von Bertalanffy and GGF $_{\text {Lo fijo }}=$ modelo de crecimiento de Gompertz con $\mathrm{L}_{0}$ fijo / Growth coefficient $\mathrm{K}$ estimated for different species of Callorhinchus genus. $\mathrm{VBGF}=$ von Bertalanffy model, $\mathrm{GGF}_{\mathrm{L} \text { fijo }}=$ Gompertz model with two parameters and fixed $\mathrm{L}_{0}$

\begin{tabular}{|c|c|c|c|c|c|c|}
\hline Especie & & $\mathrm{K}\left(\operatorname{años}^{-1}\right)$ & Modelo & Autor & Región & Método \\
\hline \multirow[t]{2}{*}{ C. milii } & M & $0,089-0,473$ & VBGF & Francis (1997) & N. Zelanda & Multifan \\
\hline & $\mathrm{H}$ & $0,06-0,224$ & VBGF & Francis (1997) & N. Zelanda & Multifan \\
\hline \multirow[t]{2}{*}{ C. milii } & M & 0,1 & VBGF & Braccini et al. (2008) & Australia & Lecturas \\
\hline & $\mathrm{H}$ & 0,09 & VBGF & Braccini et al. (2008) & Australia & Lecturas \\
\hline \multirow[t]{2}{*}{ C. capensis } & M & 0,171 & VBGF & Freer \& Griffiths (1993) & Sudáfrica & Lecturas \\
\hline & $\mathrm{H}$ & 0,0515 & VBGF & Freer \& Griffiths (1993) & Sudáfrica & Lecturas \\
\hline \multirow[t]{2}{*}{ C. callorynchus } & M & 0,473 & VBGF & Alarcón et al. (2011) & Chile & Multifan \\
\hline & $\mathrm{H}$ & 0,193 & VBGF & Alarcón et al. (2011) & Chile & Multifan \\
\hline \multirow[t]{2}{*}{ C. callorynchus } & M & 0,257 & $\mathrm{GGF}_{\mathrm{L} 0 \text { fiio }}$ & Este estudio & Argentina & Mix-ACOH \\
\hline & $\mathrm{H}$ & 0,17 & GGF $_{\text {L0 fijo }}$ & Este estudio & Argentina & Mix-ACOH \\
\hline
\end{tabular}

datos de frecuencia de tallas fue de 59 y $76 \mathrm{~cm}$ para machos y hembras, respectivamente.

Los valores del coeficiente de crecimiento obtenido en este estudio se encuentran dentro del rango de valores reportados para el género en otros estudios (Tabla 3). Las únicas estimaciones del coeficiente de crecimiento $\mathrm{K}$ que existen para la especie fueron realizadas por Alarcón et al. (2011) en el Pacífico sur en la región de Coquimbo (Chile). Los resultados de ese estudio indican que en esa región la especie tendría un crecimiento más rápido que en el Atlántico sur (GSM, Argentina). Estos autores utilizaron el programa MULTIFAN y obtuvieron un valor de $\mathrm{K}$ de 0,473 y 0,193 años ${ }^{-1}$ para machos y hembras, respectivamente. Los estudios de crecimiento a partir de estructuras de tallas con el programa MULTIFAN requieren de los mismos supuestos que el análisis con MIX (una sola época reproductiva anual, identificación clara de las cohortes en el tiempo y muestras con las primeras clases de edad bien representadas). Por lo tanto, las diferencias en los parámetros estimados en cada región deben analizarse considerando la calidad de los datos utilizados en cada estudio y por el cumplimiento de los supuestos del método. Tener representado todo el rango de tallas de la especie es importante para los análisis de crecimiento que se basan en la detección modas. El programa MULTIFAN utilizado por Alarcón et al. (2011) es una herramienta robusta donde el proceso de estimación y detección de modas se basa en un estimador de máxima verosimilitud. Estas características hacen que el método sea mucho más objetivo que el software MIX donde la detección del número de clases de edad fue determinada por inspección visual del histograma de frecuencia. Sin embargo el análisis por MIX-ACOH permitió incluir los histogramas de frecuencias de talla obtenidos en las campañas de investigación donde estaban presentes modas que representan las primeras clases de edad y que no fueron observadas en los datos de Alarcón et al. (2011). Estos autores detectaron modas para la primera clase de edad a partir de los 32,9 y $32,3 \mathrm{~cm}$ a diferencia de este estudio donde la primera clase de edad fue asignada a una longitud media de 14,53 y 13,93 cm para machos y hembras, respectivamente. Estos valores son más razonables ya que se considera que la talla de nacimiento del pez gallo es de aproximadamente $10 \mathrm{~cm}$ de LCLS ( a $15 \mathrm{~cm}$ de largo total, LT), por lo cual las longitudes medias asignadas a la primera clase de edad fueron adecuadas. Francis (1997) también evaluó el crecimiento de $C$. milii en Nueva Zelanda utilizando el método del programa MULTIFAN. Este autor comparó diferentes regiones y décadas (1960 y 1980) y obtuvo variaciones temporales en los parámetros de crecimiento $\left(\mathrm{K}_{\mathrm{y}} \mathrm{L}_{\infty}\right.$ ), lo que lo atribuyó a un incremento en la tasa de crecimiento como respuesta a la sobrepesca. Sin embargo, las diferencias reportadas podrían también deberse a la falta de representatividad de la estructura de tallas completa debido al sesgo introducido por los diferentes métodos y profundidades de muestreo en cada período. 
Otro de los supuestos del método es que las clases anuales o cohortes pueden ser identificadas en los datos de frecuencia de tallas y que el crecimiento de los individuos de cada cohorte puede ser seguido en el tiempo. Este supuesto fundamental exige que dentro del ciclo anual ocurra una sola época reproductiva y que el éxito reproductivo sea variable interanualmente (i.e., clases anuales reclutadas con abundancias diferentes). En el caso de $C$. callorynchus, la época de mayor actividad reproductiva tiende a ocurrir de agosto a noviembre, junto con la época que se observa un mayor número de hembras con cápsulas. Sin embargo, se cita reproducción durante todo el año (Di Giácomo 1990). Así, el crecimiento derivado en este estudio estará condicionado al supuesto básico de una sola época reproductiva y que los grupos de edad identificados en los datos de frecuencia de tallas son efectivamente clases anuales reclutadas con abundancia diferentes. En este contexto, los parámetros de crecimiento determinados en este estudio, deben ser considerados una hipótesis, evaluada y validada en futuros estudios por otros métodos como la lectura de edades o marcado y recaptura (Sullivan 1977, Freer \& Griffiths 1993, Francis 1997, Braccini et al. 2008). Esto se aplica también al resto de los parámetros de la historia de vida, principalmente a la tasa de mortalidad natural y longevidad teórica que están correlacionados con el coeficiente de crecimiento (K) y la talla asintótica $\left(\mathrm{L}_{\infty}\right)$.

\section{Mortalidad Y LONGEVIDAD}

Las estimaciones realizadas en este estudio indican que la especie podría alcanzar una longevidad teórica cercana a $\operatorname{los} 13$ y 21 años de edad en machos y hembras, respectivamente. En el caso de los machos estos valores son superiores a los estimados por Alarcón et al. (2011) quienes reportaron una esperanza de vida de 9,4 años para el pez gallo del Pacífico. Sin embargo, estos autores para el caso de las hembras obtuvieron una esperanza de vida de 27,9 años, mayor a la estimada en este estudio. Aunque en ambos estudios se utilizó el mismo método de estimación (Taylor 1958), las diferencias se deben a que varió el valor del parámetro $\mathrm{L}_{\infty}$ a partir del cual se estima la longevidad. Para C. milii en Nueva Zelanda estudios de marcado y recaptura indicaron que la longevidad de esta especie probablemente exceda los 20 años de edad (Francis 1997) coincidiendo con los valores estimados en este trabajo.

La mortalidad natural es generada por cualquier factor diferente a la pesca, como la depredación, las enfermedades, la falta de recursos y la muerte por edad avanzada. En el GSM, los machos presentaron una tasa de mortalidad natural mayor que en las hembras, lo que es esperable debido a la menor cantidad de clases de edad detectadas en los datos de frecuencia de tallas, la mayor tasa de crecimiento y menor longevidad respecto a las hembras. Este patrón también fue reportado para la especie en la región de Coquimbo, Chile (Alarcón et al. 2011). El rango de $M$ obtenido en este estudio fue entre 0,28 y $0,41 \mathrm{año}^{-1}$ para los machos y 0,15 y $0,30 \mathrm{año}^{-1}$ para las hembras, dependiendo de la formula empírica utilizada. En el caso de las hembras, las estimaciones de $\mathrm{M}$ del GSM fueron similares a las obtenidas por Alarcón et al. (2011) quienes estimaron una $\mathrm{M}$ entre 0,12 y $0,37 \mathrm{año}^{-1}$. Sin embargo, en los machos estos valores fueron menores a los obtenidos por Alarcón et al. (2011) en el Pacífico sudeste donde reportaron una $\mathrm{M}$ entre 0,42 y $0,82 \mathrm{año}^{-1}$. Esto se corresponde con la mayor tasa de crecimiento y menor longevidad estimada para el pez gallo en esa región respecto a la obtenida en el GSM. La mortalidad natural por falta de recursos o depredación está ligada al ecosistema donde habita la población. Una misma especie puede tener distintas tasas de mortalidad natural en diferentes zonas, según la densidad de los depredadores y competidores, que a su vez están afectados por la pesca (Sparre \& Venema 1997). Por otro lado, los métodos empíricos utilizados en este estudio asumen que la $\mathrm{M}$ es constante para todas las clases de edad. Sin embargo, los métodos que estiman $\mathrm{M}$ dependiendo de la edad pueden ser más adecuados para el caso de los condrictios, donde la $\mathrm{M}$ inicial decrece a medida que los individuos aumentan su tamaño, y únicamente se incrementa cuando llegan a su edad terminal. Estos métodos no pudieron ser utilizados ya que se requiere el parámetro de crecimiento $t_{0}$, el cual no fue estimado en este estudio, debido a que con el modelo de Gompertz seleccionado se estimó el parámetro $\mathrm{L}_{0}$. Por lo tanto, en ausencia de mejores estimaciones para la región del Atlántico la mortalidad natural estimada en este estudio puede utilizarse para futuras evaluaciones de stocks y modelos demográficos.

En consecuencia, fue demostrado en este estudio que existe dimorfismo sexual en las características de historia de vida del pez gallo en el GSM. La talla máxima, la talla de madurez y la longevidad, en el caso de las hembras, fueron mayores a la de los machos, mientras que la tasa de crecimiento y mortalidad natural fueron menores. Debido a estas diferencias, las hembras adultas podrían estar más afectadas por la presión pesquera que los machos, generando cambios demográficos en la población y el potencial de renovación del stock. 


\section{Agradecimientos}

Agradecemos a los integrantes del grupo CONDROS Téc. Matías Suarez, Téc. Gimena Mora, Lic. Verónica Jausoro y Dra. Marina Coller por la ayuda brindada en los muestreos biológicos. También queremos agradecer a la planta pesquera 'Calme - Río Salado' y a la tripulación del buque 'Viernes Santo' por proveer el material biológico y permitirnos trabajar en sus instalaciones. Este trabajo fue financiado por los proyectos MINCYT $n^{\circ} \mathrm{CH} /$ 11/05 y CONICYT n²011-645 en el marco del Programa de Cooperación Científico-Tecnológica entre el Ministerio de Ciencia, Tecnología e Innovación Productiva de la República Argentina (MINCyT) y la Comisión Nacional de Investigación Científica y Tecnológica (CONICYT) de Chile que permitió el trabajo conjunto entre los grupos de investigación.

\section{LITERATURA CITADA}

Alarcón C, L Cubillos \& E Acuña. 2011. Length-based growth, maturity and natural mortality of the cockfish Callorhinchus callorhynchus (Linnaeus, 1758) off Coquimbo, Chile. Environmental Biology of Fishes 92: 65-78.

Barnett L, R Earley, D Ebert \& G Cailliet. 2009. Maturity, fecundity, and reproductive cycle of the spotted ratfish, Hydrolagus colliei. Marine Biology 156: 301-316.

Braccini J, B Gillanders, T Walker \& J Tovar-Avila. 2007. Comparison of deterministic growth models fitted to length at- age data of the piked spurdog (Squalus megalops) in south-eastern Australia. Marine and Freshwater Research 58: 24-33.

Braccini J, T Walker \& S Conron. 2008. Evaluation of effects of targeting breeding elephant fish by recreational fishers in Western Port. Final Report to Fisheries Revenue Allocation Committee, 63 pp.

Burnham K \& D Anderson. 2002. Model selection and multimodel inference: A practical information -Theoretic approach, 488 pp. Springer, New York.

Cailliet G, W Smith, H Mollet \& K Goldman. 2006. Age and growth studies of chondrichthyan fishes: the need for consistency in terminology, verification, validation, and growth function fitting. Environmental Biology of Fishes 77: 211-228.

Chirichigno N \& R Cornejo. 2001. Catálogo comentado de los peces marinos del Perú, 314 pp. Instituto del Mar del Perú, Callao.

Cortés E. 2000. Life history patterns and correlations in sharks. Reviews in Fisheries Science 8(4): 288-344.

Crawley J. 2007. The R book, 942 pp. Wiley, West Sussex.

Di Giácomo E. 1990. Contribución al estudio biológico-pesquero del pez gallo, Callorhynchus callorhynchus (Linné, 1758) Berg, 1895, en el Golfo San Matías. Tesis Doctoral, Facultad de Ciencias Naturales y Museo, Universidad Nacional de La Plata, La Plata, 174 pp.
Di Giácomo E. 1992. Distribución de la población del pez gallo (Callorhinchus callorhynchus) en el Golfo San Matías, Argentina. Frente Marítimo 12(A): 113-118.

Di Giácomo E \& M Perier. 1991. Evaluación de la biomasa y explotación comercial del pez gallo (Callorhynchus callorhynchus) en el Golfo de San Matías, Argentina. Frente Marítimo 9: 7-13.

Di Giácomo E \& M Perier. 1994. Reproductive biology of the cockfish, Callorhynchus callorhynchus (Holocephali: Callorhynchidae), in Patagonian waters (Argentina). Fishery Bulletin 92: 531-539.

Di Giácomo E \& M Perier. 2008. Especies patagónicas para pesca a pequeña escala. Infopesca Internacional 35: 30-34.

Di Giácomo E, A Parma \& J Orensanz. 1994. Food consumption by the cock fish, Callorhinchus callorynchus (Holocephali: Callorhynchidae), from Patagonia (Argentina). Environmental Biology of Fishes 40: 199-211.

Du J. 2002. Combined algorithms for fitting finite mixture distributions. Master Science Thesis, McMaster University, Ontario, 124 pp.

Dulvy N \& R Forrest. 2010. Life histories, population dynamics, and extinction risks in chondrichthyans. In: Carrier JC, JA Musick \& MR Heithaus (eds). Sharks and their relatives II: biodiversity, adaptive, physiology, and conservation, pp. 635-676. CRC Press, Boca Raton.

Estalles M, N Coller, E Di Giácomo \& M Perier. 2011. Distribution and reproductive biology of the electric ray Discopyge tschudii Heckel, 1846 in San Matías Gulf, Northern Patagonia, Argentina. Neotropical Ichthyology 9(4): 831-838.

Figuereido J. 1977. Manual de peixes marinhos do Sudeste do Brasil. Introducao, raias e quimeras, $104 \mathrm{pp}$. Museu de Zoologia, University of Sao Paulo, Brasil.

Francis M. 1997. Spatial and temporal variation in the growth rate of elephantfish (Callorhinchus milii). New Zealand Journal of Marine and Freshwater Research 31: 9-23.

Freer D \& C Griffiths. 1993. Estimation of age and growth in the St. Joseph Callorhinchus capensis (Dumeril). South African Journal of Marine Science 13: 75-82.

Hoenig J. 1983. Empirical use of longevity data to estimate mortality rates. Fishery Bulletin 82(1): 898-902.

Jensen A. 1996. Beverton and Holt life history invariants results from optimal trade-off of reproduction and survival. Canadian Journal of Fisheries and Aquatic Sciences 53: 820822.

López H, N San Román \& E Di Giácomo. 2000. On the South Atlantic distribution of Callorhinchus callorhynchus (Holocephali: Callorhynchidae). Journal of Applied Ichthyology 16: 39.

MacDonald P \& P Green. 1988. User's guide to program MIX: an interactive program for fitting mixtures of distributions, 75 pp. Icthus Data Systems, Hamilton. 
MacDonald P \& T Pitcher. 1979. Age groups from sizefrequency data: a versatile and efficient method for analyzing distribution mixtures. Journal of Fisheries Research Board of Canada 36: 987-1001.

Moura T, I Figueiredo, P Bordalo-Machado \& L Gordo. 2004. Growth pattern and reproductive strategy of the holocephalan Chimaera monstrosa along the Portuguese continental slope. Journal of the Marine Biological Association of the United Kindom 84: 801-804.

Natanson L, N Kohler, D Ardizzone, G Cailliet, S Wintner \& H Mollet. 2006. Validated age and growth estimates for the shortfin mako, Isurus oxyrinchus, in the North Atlantic Ocean. Environmental Biology of Fishes 77: 367-383.

Pauly D. 1980. On the interrelationships between natural mortality, growth parameters and mean environmental temperature in 175 fish stocks. Journal du Conseil International Pour l' Exploration de la Mer 39(2): 175-192.

Perier M \& E Di Giácomo. 2002. El Savorín Seriolella porosa como un recurso estacional en el Golfo San Matías, República Argentina. Revista de Investigación y Desarrollo Pesquero 15: 15-26.

Perier M, M Estalles, N Coller, M Suarez, G Mora \& E Di Giácomo. 2011. Chondrichthyans of the San Matías Gulf, Patagonia, Argentina. Revista del Museo Argentino de Ciencias Naturales 13: 213-220.

R Core Team. 2012. R: A language and environment for statistical computing. R Foundation for Statistical Computing, Vienna. <http://www.R-project.org>.

Ricker W. 1975. Computation and interpretation of biological statistics of fish populations. Bulletin of the Fisheries Research Board of Canada 191: 1-382.

Rickhter V \& V Efanov. 1976. On one of the approaches to estimation of natural mortality of fish populations. Report No. 76/VI/8 International Commission of the Northwest Atlantic Fisheries.

Roa R. 1993. Annual growth and maturity function of the squat lobster Pleuroncodes monodon in central Chile. Marine Ecology Progress Series 97: 157-166.

Roa R \& B Ernst. 1996. Age structure, annual growth, and variance of size at age of the shrimp Heterocarpus reedi. Marine Ecology Progress Series 137: 59-70.
Roa R, B Ernst \& F Tapia. 1999. Estimation of size at sexual maturity: an evaluation of analytical and resampling procedures. Fishery Bulletin 97: 570-580.

Romine J, R Grubbs \& J Musick. 2006. Age and growth of the sandbar shark, Carcharhinus plumbeus, in Hawaiian waters through vertebral analysis. Environmental Biology of Fishes 77: 229-239.

Sánchez R, G Navarro, E Calvo \& F del Castillo. 2011. La pesca y comercialización de condrictios en Argentina. Dirección Nacional de Planificación Pesquera. En: Wöhler O, P Cedrola \& M Cousseau (eds). Contribuciones sobre la biología, pesca y comercialización de tiburones en la Argentina. Aportes para la elaboración del Plan de Acción Nacional, pp. 151-184. Consejo Federal Pesquero, Buenos Aires.

Sánchez, R, G Navarro \& V Rozycki. 2012. Estadísticas de la pesca marina en la Argentina. Evolución de los desembarques 1898-2010, 528 pp. Ministerio de Agricultura, Ganadería y Pesca de la Nación, Buenos Aires.

Simpfendorfer C, R Bonfil \& J Robert \& R Latour. 2005. Mortality estimation. In: Musick J \& R Bonfil (eds). Management techniques for elasmobranch fisheries. FAO Fisheries Technical Paper 474: 166-185.

Sparre P \& S Venema. 1997. Introducción a la evaluación de recursos pesqueros tropicales. Parte 1. Manual. FAO Documento Técnico de Pesca 306(1) Rev. 2: 1-420.

Sullivan K. 1977. Age and growth of the Elephant fish Callorhinchus milii (Elasmobranchii: Callorhynchidae). New Zealand Journal of Marine and Freshwater Research 11(4): 745-753.

Taylor C. 1958. Cod growth and temperature. Journal du Conseil International Pour l' Exploration de la Mer 23: 366-370.

Venables W \& B Ripley. 2002. Modern applied statistics with S, 498 pp. Springer, New York.

Von Bertalanffy L. 1938. A quantitative theory of organic growth. Human Biology 10: 181-213.

Recibido el 12 de septiembre de 2014 y aceptado el 23 de marzo de 2015

Editor: Claudia Bustos D. 